\title{
CALCULO DE FLUJOS DE CALOR EN LA INTERFASE AIRE-AGUA DEL LAGO
} DE BANYOLES.

\section{J. Colomer y X. Casamitjana}

Institut d'Ecologia Aquatica. Universitat de Girona. Pl. Hospital, 6. 17071 Girona.

Palabras clave: Flujos de calor, radiación de onda corta, radiación de onda larga, albedo, evaporación, calor sensible, lago de Banyoles.

\section{ABSTRACT}

The surface heat balance of a lake is govemed by shortwave (solar) and longwave (from sky and terrestrial) radiation, latent heat, sensible heat and to a far less extent by energy associated with the inflows and outflows (Holtslag \& Van Ulden, 1983; Henderson-Sellers, 1986 ; Marti \& Imboden, 1986; Livingstone \& Imboden, 1989). In this paper we calculate the surface fluxes in lake Banyoles and we relate them to routinely observed meteorological parameters such as air temperature, relative humidity, wind velocity and cloud cover, and to limnological parameters such as water surface temperature, inflows and outflows. Then, a simple empirical scheme is presented, which gives estimates of the surface fluxes at hourly and daily temporal scales.

\section{INTRODUCCION}

La determinación de los flujos de calor en la interfase aire-agua del lago de Banyoles, incorpora las radiaciones de onda corta (solar) y de onda larga (terrestre y atmosférica) junto a los flujos de evaporación/condensación (calor latente) y conducción (calor sensible).

En este trabajo, se han analizado varios modelos predictivos de las magnitudes anteriores y se han propuesto diferentes modificaciones de las ecuaciones que calculan estos flujos de calor. Se presenta, por tanto, un conjunto de ecuaciones que componen un modelo semiexperimental de predicción de los flujos de calor en la superficie de un lago situado en un clima mediterráneo.

El análisis y cuantificación de estos flujos es de gran interés ya que permiten conocer las condiciones de la capa límite atmosférica, aparición o destrucción de la termoclina, etc. Estos flujos son necesarios a su vez para plantear tanto modelos hidrodinámicos como modelos de predicción climatológicos o de transporte de contaminantes.

\section{MATERIAL Y METODOS}

Los flujos energéticos se han evaluado a partir de la medición de parámetros atmosféricos tales como la radiación solar global, la radiación atmosférica neta, la temperatura del aire y del agua, la presión atmosférica, la velocidad del viento, la humedad relativa y el "cloud cover". Estas medidas se han llevado a cabo durante 21 meses, desde marzo de 1989 hasta noviembre de 1990.

Los datos meteorológicos utilizados para la elaboración del cálculo de flujos de calor han sido registrados por una estación meteorológica de tipo automático, que dispone de 11 sensores, que se encuentra a 100 metros del lago de Banyoles, a $12 \mathrm{~m}$ sobre el nivel del mismo. El intervalo de medida ha variado entre 20 y 30 minutos. También se han realizado medidas de la temperatura superficial del lago de Banyoles así como de los influentes y efluentes. En las fig. $1 \mathrm{a}, \mathrm{b}$ y $\mathrm{c}$ presentamos la evolución durante todo el período de estudio de la temperatura media diaria del aire, $\mathrm{T}_{\mathrm{a}}$, de la humedad relativa media diaria del aire, $H R$, y de la temperatura media diaria del agua, $T_{w}$.

\section{RESULTADOS Y DISCUSION}

El balance neto de calor en la superficie de un lago puede evaluarse a partir de 5 procesos principales de transferencia de energía en la interfase aire-agua. La ecuación que rige este balance es

$$
\mathrm{Q}=\mathrm{K}^{\downarrow}\left(1-\mathrm{AS}_{\mathrm{S}}\right)+\mathrm{L} \downarrow\left(1-\mathrm{A}_{\mathrm{L}}\right)+\mathrm{L}^{\uparrow}+\mathrm{HE}_{\mathrm{E}}+\mathrm{H}_{\mathrm{C}}
$$



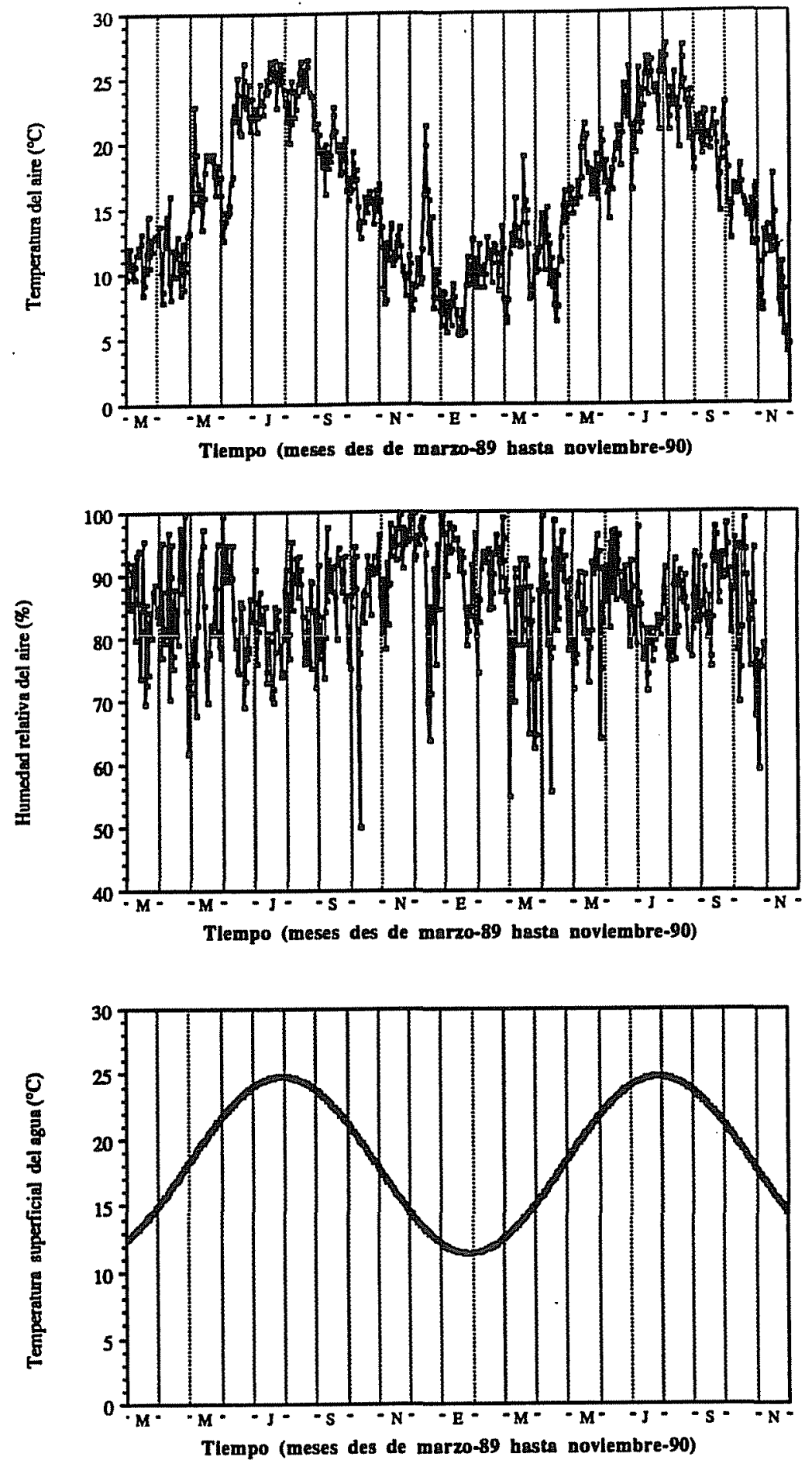

Figura 1. Evolución temporal de tres parámetros durante el periodo de estudio: marzo -89 hasta noviembre -90 . (a) Temperatura del aire, (b) Humedad relativa del aire y (c) Temperatura del agua. 
donde $\mathrm{K} \downarrow$ es la radiación solar incidente global (difusa y directa) de onda corta , $\mathrm{L} \downarrow$ es la radiación atmosférica incidente de onda larga, As y $A_{L}$ son las reflexiones (albedos) de onda corta y larga respectivamente, $\mathrm{L} \uparrow$ es la radiación de onda larga emitida por la superficie del lago hacia la atmosfera, $\mathrm{H}_{\mathrm{E}}$ es el flujo de calor latente intercambiado entre la superficie del lago y la atmósfera debido a los procesos de evaporación y condensación, $\mathrm{H}_{C}$ es el flujo de calor sensible intercambiado entre la superficie y la atmósfera y $Q$ es la cantidad de calor neto que se transfiere al lago. $Q$ será positivo cuando la superficie del lago gane calor y $Q$ será negativo cuando la superficie pierda calor.

\section{Modelo de onda corta}

El modelo de radiación de onda corta que se propone en este trabajo parametriza la radiación solar incidente que se recibe en la superficie del lago de Banyoles en función de la elevación solar, del "cloud cover" y de la reflexión o albedo de onda corta. Se propone un conjunto de ecuaciones que tienen en cuenta los principales factores locales como latitud, declinación solar, coeficientes de turbiedad y reflexión superficial. Los coeficientes de estas ecuaciones se obtienen ajustando los valores teóricos a los experimentales obtenidos en la estación meteorológica del lago de Banyoles. Las ecuaciones que componen el modelo son:

Flujo de radiación de onda corta

$$
\mathrm{K} \downarrow(1-\mathrm{AS})
$$

Flujo de radiación de onda corta (solar) en ausencia total de nubes (modelo horario)

$$
\mathrm{K}_{\mathrm{o}}=\mathrm{a}_{1} \operatorname{sen} \emptyset+\mathrm{a}_{2}
$$

donde $\emptyset$ es la elevación solar y $a_{1}$ y a son coeficientes empíricos: $a_{1}=1118.18$ y $a_{2}=-84.76$

$\operatorname{sen} \emptyset=\operatorname{sen} \psi \operatorname{sen} \delta+\cos \psi \cos \delta \cos h$

$\delta=\operatorname{arcsen}(0,398$ sen (SL))

$\mathrm{SL}=4.871+0.0175 \mathrm{D}+0.033$ sen $(0.0175 \mathrm{D})$

donde $\psi$ es la latitud, $\delta$ es la declinación solar y h es el ángulo horario.

Flujo de radiación de onda corta (solar) en ausencia total de nubes (modelo diario)

donde $\mathrm{D}$ es el día.

$$
K_{0}=476.64-253.35 \operatorname{sen}(\pi(D+85) / 183)
$$

Flujo de radiación de onda corta (solar) en presencia total de nubes (modelo diario)

$$
K_{1}=115.13-80.16 \operatorname{sen}(\pi(D+75) / 183)
$$

Indice de cubrimiento del cielo: Cloud cover

Para calcular el índice diario de cubrimiento del cielo, $\mathrm{C}$, hemos interpolado los valores diarios de $\mathrm{K} \downarrow$ medidos en la estación meteorológica, a partir de la curva de radiación solar con índice de cubrimiento cero, ecuación (4) y la curva de radiación solar con índice de cubrimiento 1, ecuación (5).

Flujo de radiación de onda corta (solar) en presencia de nubes (modelo diario)

$$
\mathrm{K} \downarrow=\mathrm{K}_{\mathrm{o}}\left(1+\mathrm{c}_{1} \mathrm{C} \mathrm{c} 2\right)
$$

donde $K_{0}$ es el valor de la radiación solar sin nubes, ec. (4) y $c_{1}$ y $c_{2}$ son coeficientes empíricos: $c_{1}$ $=-0.707$ y $c_{2}=1.03$ 


$$
\begin{gathered}
\text { Albedo de onda corta } \\
\mathrm{A}_{8}(\mathrm{t})=\frac{\mathrm{a}_{0}}{\mathrm{a}_{0}+\sin \phi} \\
\mathrm{a}_{0}=0.02+0.01(0.5-\mathrm{C})(1-\operatorname{sen}(\pi(\mathrm{D}-81) / 183)
\end{gathered}
$$

En la fig. 2 presentamos la evolución de la radiación solar diaria $\mathrm{K} \downarrow$ medida en la estación meteorológica y en la fig. 3 se presenta la comparación entre los valores experimentales medidos y los valores estimados por el modelo que aqư proponemos.

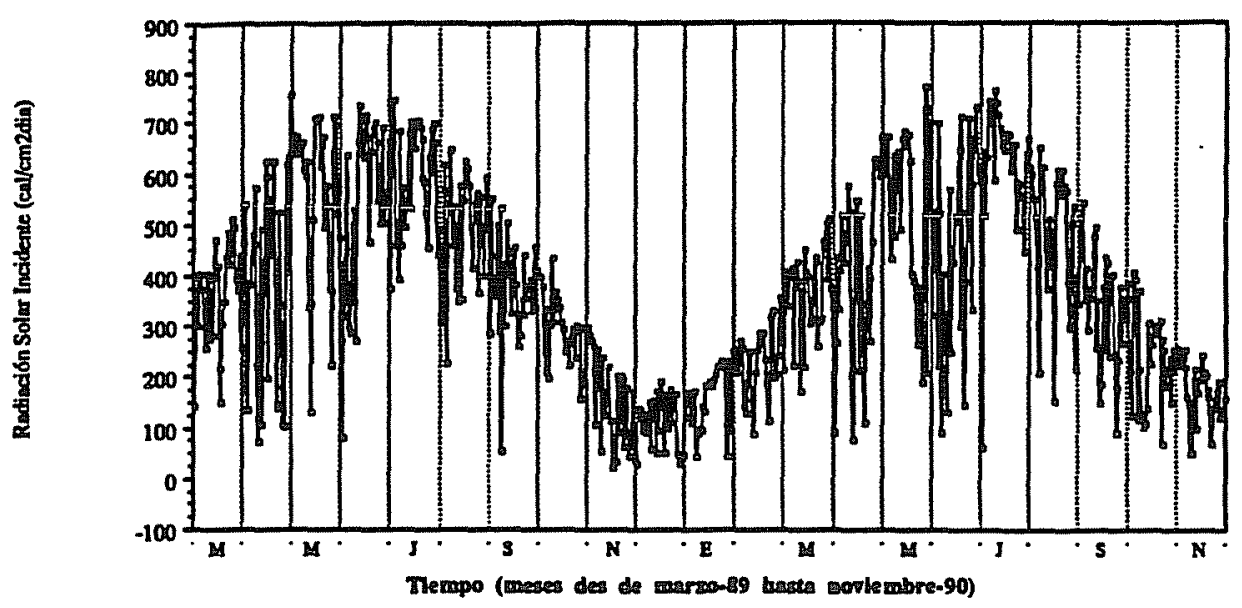

Figura 2. Evolución temporal de la radiación solar incidente en la superficie del lago, medida en la estación meteorológica, durante el periodo de estudio.

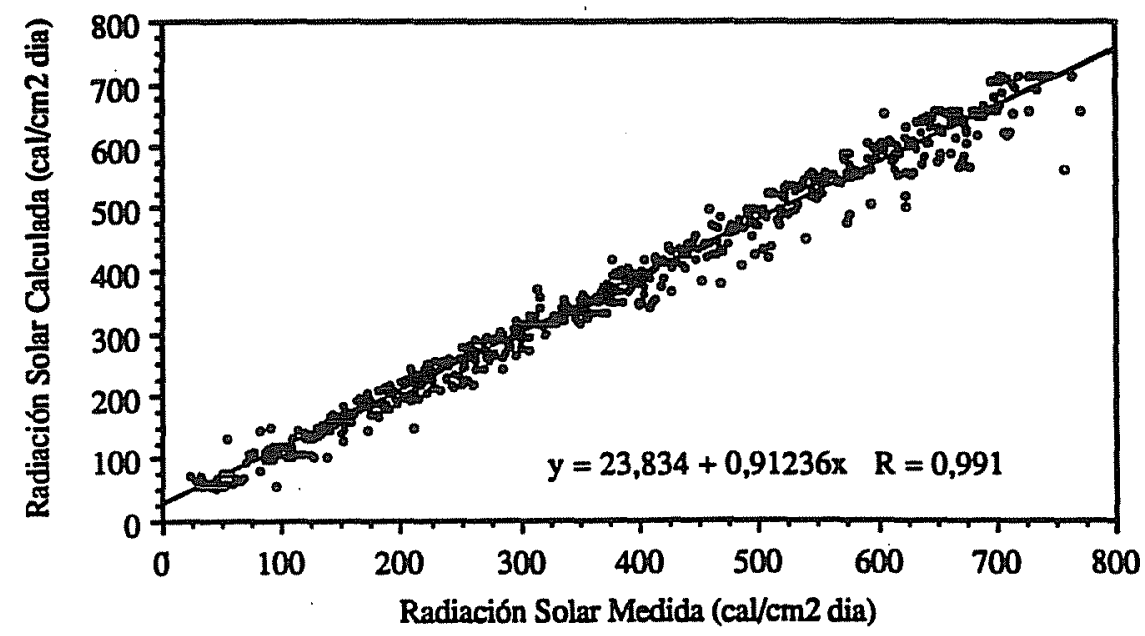

Figura 3. Comparación entre los valores diarios de la radiación solar calculados según las ecuaciones propuestas en el modelo y los.valores diarios medidos experimentalmente en la estación meteorológica. 


\section{Modelo de onda larga}

El modelo de radiación de onda larga incorpora la radiación de ona larga que proviene de la atmósfera y la radiación de ona larga emitida por la superficie del lago. La radiación incidente atmosférica se calcula a partir de la ley de Stefan-Boltzmann corregida por la emisividad atmosférica debido a que la atmósfera, así como la superfície del lago, se consideran cuerpos grises. Los coeficientes propuestos en la ecuación de la emisividad atmosférica en ausencia de nubes (11) implican una correlación mayor entre los valores experimentales y los teóricos. El albedo de onda larga es un parámetro que se considera constante, 0.03 , con lo que la emisividad de la superficie del agua valdrá 0.97. Las ecuaciones que componen este modelo son :

\section{Flujo de radiación de onda larga}

$$
\mathrm{L} \downarrow(1-\mathrm{AL})
$$

Flujo de radiación de onda larga en ausencia total de nubes

$$
\begin{gathered}
L_{0}=\varepsilon \sigma T_{a}{ }^{4} \\
\varepsilon=0.57+9.2610^{-7} e_{a} \exp \left(1500 / T_{a}\right)
\end{gathered}
$$

Flujo de radiación de onda larga en presencia de nubes

$$
\begin{gathered}
L^{\downarrow}=\varepsilon^{\prime} \sigma T_{\mathrm{a}^{4}} \\
\varepsilon^{\prime}=\varepsilon\left(1+\mathrm{K} \mathrm{C}^{2}\right) \quad \text { donde } \mathrm{K}=0.17
\end{gathered}
$$

Albedo de onda larga

$$
A_{L}=0.03
$$

Flujo de radiación de onda larga emitida por la superficie del lago

$$
L \uparrow=-0.97 \sigma T_{w}^{4}
$$

$$
T_{w}=291.1-6.7 \operatorname{sen}(\pi(D+65) / 183)
$$

\section{Calor latente y calor sensible}

Los flujos de calor latente (evaporación) y calor sensible (conducción) se consideran en la misma sección ya que el calor sensible perdido en la interfase aire-agua del lago se relaciona con el calor latente por la relación de Bowen. Estos flujos se pueden deducir a partir de la teoría semiempírica de turbulencia (Livingstone \& Imboden, 1989). Las ecuaciones que rigen estos dos procesos son:

Flujo de calor latente y sensible

$$
\begin{gathered}
H_{E}=-f(u)\left(e_{s}-e_{a}\right) \\
f(u)=4.8+1.92 u_{12}+0.28\left(T_{s}-T_{a}\right) \\
H_{C}=H_{E} 0.63\left(T_{s}-T_{a}\right) /\left(e_{s}-e_{a}\right)
\end{gathered}
$$


donde $f(u)$ es un función que depende de la velocidad del viento, $u_{12}, y$ de las temperaturas del aire $y$ del agua. Los valores de $e_{s}$ y $e_{a}$ son a su vez las presiones de vapor a la temperatura superficial del agua del lago y a la temperatura del aire.

En la figura 4 presentamos la evolución de cada uno de los flujos de calor, las magnitudes de los cuales difieren bastante. También podemos observar el desfase que hay entre las curvas de tipo senoidal. Los dos procesos que más contribuyen al valor del flujo total superficial son la emisión y absorción de onda larga. El valor medio de la radiación que emite la superficie del lago durante el periodo de estudio es $-830 \mathrm{cal} \mathrm{cm}^{-2}$ dia-1, $^{-1}$ y el de la absorción de onda larga es de $694 \mathrm{cal} \mathrm{cm}^{-2} \mathrm{dia}^{-1}$. Por tanto, la suma de estos dos términos o radiación de onda larga efectiva da siempre un valor negativo, cuyo valor medio es $-136 \mathrm{cal} \mathrm{cm}^{-2}$ dia-1. Ya que los valores de absorción y emisión de onda larga son aproximadamente iguales y de signo contrario y presentan un desfase de $180^{\circ}$, los valores de radiación de onda corta juegan un papel muy importante en la determinación de los flujos superficiales. Por tanto, la suma de los tres flujos radiativos, absorción de ona larga, emisión de onda larga y absorción de onda corta (solar), presenta la misma variación senoidal que la radiación de onda corta.

La suma de los flujos no radiativos, calor latente y calor sensible es aproximadamente 5 veces menor que la suma de los flujos radiativos. Por otra parte, el calor que pierde el lago por los procesos de evaporación y condensación (calor latente) es aproximadamente cuatro veces mayor que el calor perdido por procesos de conducción (calor sensible). Además, el desfase entre los flujos radiativos y no radiativos da lugar a que mientras la radiación de onda corta alcanza los valores máximos durante el mes de junio (solsticio), la suma de los flujos superficiales alcanza los valores máximos durante los meses de julio o agosto.

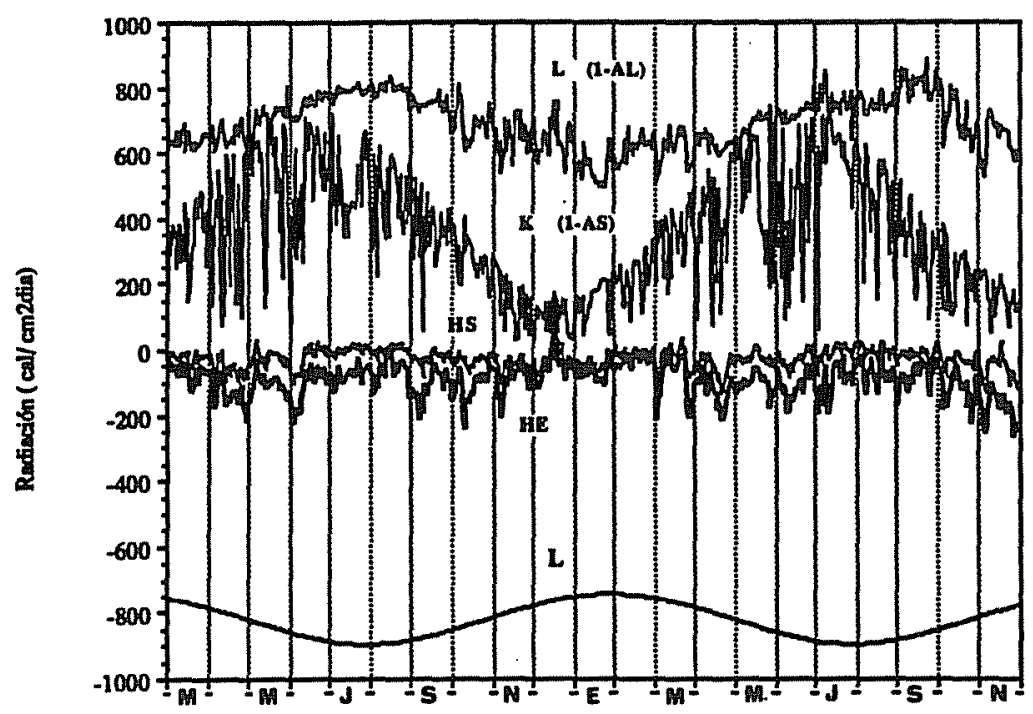

Thempo (meses desde marzo-89 hasta noviembre-90)

Figura 4. Evolución temporal de los 5 flujos superficiales de transferencia de energía en la interfase aire-agua del lago de Banyoles. 


\section{BIBLIOGRAFIA}

Henderson-Sellers, B.. 1986. Calculating the surface energy balance for lake and reservoir modeling: a review. Reviews of geophisics, 24, 3. 625-649.

Holtslag, A.M. \& A.P.Van Ulden. 1983. A simple scheme for daytime estimates of the surface fluxes from routine weather data. Journal of climate and applied meteorology, 22, 4. 517-529.

Livingstone, D,M. \& D.M. Imboden. 1989. Annual heat balance and equilibrium temperature of Lake Aegeri, Switzerland. Aquatic Sciences, 51/4. 351-370.

Marti, D.E. \& D.M. Imboden. 1986. Thermische energieflüsse an der wasseroberfläche: Beispiel Semparchersee. Schweiz. Z. Hydrol, 48 (2). 196-229. 